\title{
PROVISIONAL CHECKLIST FOR THE LICHENS OF CHIAPAS
}

\author{
HARRIE J. M. Sipman \\ Botanischer Garten und Botanisches Museum \\ Königin-Luise-Str. 6-8 \\ D-14191 Berlin \\ Germany \\ AND \\ JAN H. D. WOLF \\ El Colegio de la Frontera Sur (ECOSUR) \\ Apartado postal 63 \\ 29290 San Cristóbal de Las Casas \\ Chiapas, México
}

\begin{abstract}
A list is presented of 359 lichen taxa known so far from Chiapas (Mexico). It is based on literature records and recent collections made by Breedlove and by Wolf and collaborators. The actual lichen flora of Chiapas is estimated to be certainly much larger.
\end{abstract}

\section{RESUMEN}

Se presenta una lista preliminar de 359 taxa de líquenes del estado de Chiapas, México, basada en datos de la literatura y colectas recientes de Breedlove y de Wolf y sus colaboradores. Se estima que el número total de especies para el estado es considerablemente más grande.

\section{INTRODUCTION}

The southernmost state of Mexico, Chiapas, is diverse in soils, climate and associated forest formations. These range from semi-desert vegetation to rainforests and from lowland mangroves to sub-alpine vegetation at the up to $4,000 \mathrm{~m}$ high mountain peaks. Not surprisingly, the state of Chiapas is considered to be among the richest in species of the country, despite of its small territory of slightly less than $4 \%$ of the whole country. For example, Chiapas occupies the second place regarding vertebrates endemic to Mesoamerica, some 875 species. The number of vascular plant species recorded for Chiapas is 8,248 species, of an estimated total of 22,800 for the whole country (Breedlove 1981, 1986; Rzedowski, 1992; Flores V. \& Gerez, 1994). 
For the bryophytes also, the state is considered as one of the potentially richest of Mexico (Delgadillo \& Cárdenas S., 1989). According to these authors, its high species diversity depends not only on its high habitat diversity, but also on its geographical position at the junction of several presumed pathways of floristic migration and on the many tropical lowland taxa which reach their northern limit in the state. Lichens may well serve to corroborate hypotheses on the past migration routes of mosses, since both groups of organisms share many habitats and have similar dispersal mechanisms.

Fortunately the luxuriant lichen growth in Chiapas has attracted the attention of lichen specialists for several decades. This resulted in the description of a number of new taxa, and the inclusion of Chiapas material in several taxonomic revisions. However, the published information is restricted to scattered records, and gives no indication of the composition and diversity of the flora, its zonation or its geographical distribution. It is certainly still a long way to a good knowledge of these aspects, but it seemed appropriate as a stimulus for further investigations, to collect all currently available information and present a first survey of the lichen flora. This including locality data, when available, in view of the state's interesting phytogeographical position.

Lichen collecting in Chiapas appears to be a remarkably recent feature. While the oldest information about the lichen flora of the Neotropics, including Mexico (Godínez \& Ortega, 1989), goes back to the earliest years of systematics, nothing could be found about lichens from Chiapas before 1925, when the first lichen collections were made by E. Matuda. No survey of the lichen flora has been published so far. For Mexico as a whole, a checklist was published by Imshaug (1956), but this is without state indications. A new, draft checklist of Mexico, with state indications, is available on Internet, offered by B. D. Ryan, T. H. Nash III \& M. Herrera Campos, at the address <http://lichen.la.asu.edu/sonoran.desert/chekmex.html>.

The first extensive lichen collections were made by Mason E. Hale Jr., lichenologist at US. He travelled in Chiapas together with T. R. Soderstrom in March 1960. His collections, probably several hundred specimens, are kept in US and are particularly rich in Parmeliaceae. The most important of the later lichen collectors are probably K. Kalb \& G. Plöbst (January 1979, probably several hundred specimens, in hb. Kalb), D. E. Breedlove with collaborators, J. Strother, B. Bartholomew, F. Almeda, M. Bourell and T. F. Daniel (197288, several hundred specimens, in CAS, large set of duplicates in B), W. \& C. Culberson (c. 1970-1990, probably several hundred specimens, in DUKE), J. Wolf \& H. Sipman (1994, c. 500 specimens, in ECOSUR, large set of duplicates in B) and J. Wolf \& F. Chanona (1997, c. 100 specimens, in ECOSUR, large set of duplicates in B). Apparently smaller numbers of collections were made by F. Almeda (specimens in CAS), E. Matuda (1925-37), specimens in $\mathrm{MICH}$ and TNS), A. J. Sharp (1945, first set of specimens in TENN?) and S. S. Tillet (1963, specimens in US). Among the Mexican botanists who explored the lichen flora of the state, the foremost to be mentioned is G. Guzmán (large set of specimens in ENCB).

Several new species were described from these collections, mostly by M. Hale and collaborators: Anaptychia polyrhiza, Everniastrum lipidiferum, Graphis glaucopsis, G. stygioarachnoides, Heterodermia lutescens, H. subisidiosa, Parmotrema chiapense, P. mesogenes, P. myelochroum, P. viridiflavum, Phaeographina elliptica, P. strigops, Ramalina asahinae and Rimeliella neotropica. This might suggest a flora rich in endemics. However, most of the macrolichen species of this list have turned out to be widespread in the Neotropics, while the included microlichens belong to Graphidaceae, still a poorly known group with uncertain species distributions. Consequently the real number of endemic lichen 
species in Chiapas is probably very low, a pattern also observed in the high-elevation mosses of this state (Delgadillo \& Cárdenas S., 1989).

The survey presented below is primarily based on the literature. Some literature records might have escaped our attention, in particular when the state was not indicated. Where only a locality name is given, it may be difficult to decide if it is in Chiapas, because the same locality names are often used for various places in Mexico. For instance, Liebmann's collections from Palenque, treated by Vainio (1926) and other lichenologists, turned out not to be from the site with the famous ruins in Chiapas. His locality is situated in Veracruz, between Veracruz and Colipa, where he was in March 1841 (McVaugh 1987: 8). Similarly we suppose that collections by J. W. Eckfeldt from "Monterey in Mexico" were not from Chiapas.

Since the available literature deals mainly with selected taxa, an effort was made towards a more representative survey by adding information from unpublished collections. For this purpose results were available from fieldwork by the authors, aimed at representative sampling of the lichen flora at selected sites (see Table 1). In addition, a considerable number of specimens collected by Breedlove were available for study, originating from a much wider range of localities, but largely restricted to macrolichens.

Table 1. List of collecting localities of Wolf \& Sipman 1994 (1-12) and Wolf \& Chanona 1997 (13).

(1) 22 Nov. 1994. Mpio. San Cristóbal de las Casas, Estación Biológica Huitepec. 1644'N, 92²41'W. $2300 \mathrm{~m}$. Región Los Altos. Bosque abierto de encino; bosque maduro de encino.

(2) 10 April, 23 Nov. 1994. Mpio. Huixtán, Rancho Merced Bazom. 1644'N, 92²9'W. 2400 m. Región Los Altos. Bosque de Pinus y Quercus.

(3) 24 Nov. 1994. Mpio. Huixtán, Rancho El Porvenir, ca. 3 km adelante de El Escalón. 1642'N, $92^{\circ} 30^{\prime} W .2400$ m. Región Los Altos. Bosque de neblina con Quercus y Pinus; potrero.

(4) 20 Mar., 25 Nov. 1994. Mpio. San Cristóbal de las Casas, Fracc. La Cañada, al Periférico Sur de la ciudad de San Cristóbal. 16²'N, 92³9'W. 2350 m. Región Los Altos. Bosque de Pinus y Quercus; potrero con Crataegus pubescens.

(5) 29 Nov. 1994. Mpio. La Trinitaria, Parque Nacional Lagunas de Montebello, Paso del Soldado. $16^{\circ} 07^{\prime} \mathrm{N}, 9^{\circ} 43^{\prime} \mathrm{W} .1500 \mathrm{~m}$. Bosque de Pinus maximinoi y Quercus sapotifolia; exposición N; bosque perturbado.

(6) 1 Dec. 1994. Mpio. Chiapa de Corzo, El Chorreadero 1645'N, 9258'W. 900 m. Región Depresión Central. Bosque mesófilo en fondo de cañón con Brosimum alicastrum dominante.

(7) 1 Dec. 1994. Mpio. Ocozocoautla, c. $5 \mathrm{~km}$ antes del aeropuerto San Juan en la carretera 190, Tuxtla Gutiérrez-México. 16²4'N, 93¹6'W. 1200 m. Región Depresión Central. Bosque caducifolio bajo seco sobre roca calcárea.

(10) 20 Oct. 1994. Mpio. San Cristóbal de las Casas, El Chivero, Los Cerezos. 16²4'N, 93³1'W. 2400 m. Región Los Altos. Bosque maduro de Pinus spp. y Quercus spp.

(11) 9 Jun. 1994. Mpio. San Cristóbal de las Casas, Paraje: art. 115 constitucional; camino de Herradura a Corralito. $16^{\circ} 42^{\prime}$ N, $92^{\circ} 37^{\prime}$ W. 2274 m. Región Los Altos. Bosque perturbado de Pinus oaxacana y Quercus segoviensis, abierto por extracción de leña.

(12) 22 Jun. 1994. Mpio. San Cristóbal de las Casas, Mitzitón, paraje: Chac' tajal. 1640'N, 92³2'W. 2405 m. Región Los Altos. Bosque de Pinus perturbado con Pinus tecunumanii dominante.

(13) 16 Jan.-13 Dec. 1997. Mpio. Ocozocoautla, c. $5 \mathrm{~km}$ antes del aeropuerto San Juan en la carretera 190, Tuxtla Gutiérrez-México. $16^{\circ} 45^{\prime} \mathrm{N}, 93^{\circ} 16^{\prime} \mathrm{W} .1200$ m. Región Depresión Central. Bosque caducifolio bajo seco sobre roca calcárea. 
The high number of species represented by single specimens suggests that most species are known by incidental findings only, and that the lichen flora of Chiapas is very incompletely represented. This suggestion is supported by the high numbers of still unidentified specimens in the collections of Wolf \& Sipman: $56 \%$. Evidently the lichen flora of Chiapas is at present very incompletely known, and certainly more than twice as many species should be expected. With the publication of this checklist, we hope to stimulate further research in the diverse lichen flora of Chiapas.

Annotated list of species, with synonyms

This list comprises 359 accepted species. Included are synonyms and infraspecific taxa, as far as they have been published from Chiapas, locality and habitat information and specimen distribution when available. Added are recent collections made by Breedlove and Wolf and their collaborators. For the Wolf's collections, locality and habitat are indicated by a number in brackets, which is explained in Table 1. Unless otherwise indicated, the cited specimens are deposited as follows: leg. Breedlove (et al.) in CAS; leg. Wolf (et al.) in $\mathrm{B}$ and in the herbarium at ECOSUR (ECO-SC-BL).

Anaptychia barbifera (Nyl.) Trev. = Heterodermia barbifera.

Anaptychia casarettiana Massal. = Heterodermia casarettiana.

Anaptychia comosa (Eschw.) Massal. = Heterodermia comosa.

Anaptychia corallophora auct., non (Tayl.) Vain. = Heterodermia crocea.

Anaptychia dissecta Kurok. var. koyana Kurok. = Heterodermia dissecta.

Anaptychia domingensis (Ach.) Massal. = Heterodermia albicans.

Anaptychia flabellata (Fée) Massal. var. flabellata = Heterodermia flabellata var. flabellata.

Anaptychia granulifera (Ach.) Massal. = Heterodermia granulifera.

Anaptychia isidiophora (Nyl.) Vain. = Heterodermia isidiophora.

Anaptychia leucomelaena fo. albociliata (Nyl.) Hue = Heterodermia leucomelos ssp. leucomelos.

Anaptychia leucomelaena (L.) Massal. fo. leucomelaena = Heterodermia leucomelos ssp. leucomelos.

Anaptychia leucomelaena fo. palmiformis Kurok. = Heterodermia leucomelos ssp. leucomelos.

Anaptychia leucomelaena fo. verrucifera Kurok. = Heterodermia verrucifera.

Anaptychia lutescens Kurok. = Heterodermia lutescens.

Anaptychia magellanica Zahlbr. var. pectinata (Zahlbr.) Kurok. fo. pectinata = Heterodermia magellanica.

Anaptychia magellanica Zahlbr. var. pectinata (Zahlbr.) Kurok. fo. subisidiosa Kurok. = Heterodermia subisidiosa.

Anaptychia obscurata (Nyl.) Vain. = Heterodermia obscurata.

Anaptychia polyrhiza Kurok. = Heterodermia polyrhiza.

Anaptychia pseudospeciosa Kurok. var. tremulans (Müll. Arg.) Kurok. = Heterodermia speciosa.

Anaptychia squamulosa Degel. = Heterodermia squamulosa. 
Anaptychia subisidiosa (Kurok.) Kurok. = Heterodermia subisidiosa.

Anaptychia tropica Kurok. var. tropica = Heterodermia tropica.

Anaptychia tropica var. antillarum (Vain.) Kurok. = Heterodermia antillarum.

Anisomeridium subprostans (Nyl.) R. C. Harris.

(7) Wolf \& Sipman 2289.

Anzia cf. masonii Yoshim.

(3) Wolf \& Sipman 1952. This seems to be the same species as Anzia sp. treated by Yoshimura (1995: 385).

Arthonia cinnabarina (DC.) Wallr.

El Sumidero Cañón, Kalb \& Plöbst s.n. (Kalb 1983: 3, as A. tumidula). - (1) Wolf \& Sipman 1800; (3) Wolf \& Sipman 2002; (5) Wolf \& Sipman 2111; (7) Wolf \& Sipman 2204, 2227.

Arthonia tumidula (Ach.) Ach. = A. cinnabarina.

Arthrorhaphis citrinella (Ach.) Poelt

Breedlove \& Bourell 67362A.

Baeomyces rufus (Huds.) Rebent.

Breedlove 57087.

Brigantiaea leucoxantha (Spreng.) R. Sant. \& Haf.

(1) Wolf \& Sipman 1799; (3) Wolf \& Sipman 1955; (5) Wolf \& Sipman 2086 [ECOSUR].

Buellia coccinea (Fée) Aptroot

(5) Wolf \& Sipman 2115a; (6) Wolf \& Sipman 2170.

Buellia lauricassiae (Fée) Müll. Arg.

(7) Wolf \& Sipman 2201.

Buellia rufofuscescens Vain.

(5) Wolf \& Sipman 2115.

Buellia trachyspora Vain.

(7) Wolf \& Sipman 2276.

Buelliella minimula (Tuck.) Fink ex Hafellner

Breedlove \& Bourell 67469; on Pertusaria sp.

Bulbothrix coronata (Fée) Hale

Hale 20827 [US] (Hale 1976: 14).

Bulbothrix isidiza (Nyl.) Hale

Hale 20170, 20184 [US] (Hale 1976: 18).

Bulbothrix tabacina (Mont. \& v.d. Bosch) Hale

Hale 21067 [US], 20122 [S, US] (Hale 1976: 24). — Breedlove 67128 [CAS], det. H. Sipman.

Bulbothrix ventricosa (Hale \& Kurok.) Hale

Near ranch, El Suspiro, Hale 20166 [US] (Hale \& Kurokawa 1964: 140, as Parmelia;

Hale 1976: 26).

Bunodophoron melanocarpum (Sw.) Wedin

(3) Wolf \& Sipman 1980, 1995; Breedlove 58190.

Byssoloma chlorinum (Vain.) Zahlbr.

(2) Wolf \& Sipman 1826.

Calicium chlorosporum F. Wils.

Lagunas de Montebello, Kalb \& Plöbst S184 (Tibell \& Kalb 1992: 17; Tibell 1996: 18). 
Calicium glaucellum Ach.

10 km NW of Comitán de Domínguez, Kalb \& Plöbst S234 (Tibell \& Kalb 1992: 19; Tibell 1996: 19).

Calicium hyperelloides $\mathrm{Nyl}$.

Lagunas de Montebello, Kalb \& Plöbst S178; 10 km NW of Comitán de Domínguez, Kalb \& Plöbst S223; id., 20 km, S176; id., 25 km, S179; close to Jitotol on Hwy. 195, Kalb \& Plöbst S175, S227 (Tibell \& Kalb 1992: 22; Tibell 1996: 21).

Calicium lenticulare Ach.

Lagunas de Montebello, Kalb \& Plöbst S193 (Tibell \& Kalb 1992: 26; Tibell 1996: 23).

Calicium salicinum Pers.

20 km NW of Comitán de Domínguez, Kalb \& Plöbst S207; id., 25 km, S209; 30 km SW of Palenque, Kalb \& Plöbst S208 (Tibell \& Kalb 1992: 33; Tibell 1996: 26).

Calicium tricolor F. Wils.

Lagunas de Montebello, Kalb \& Plöbst s.n., S241 (Kalb 1991: 7; Tibell \& Kalb 1992: 35; Tibell 1996: 28). — (5) Wolf \& Sipman 2078.

Caloplaca brebissonii (Fée) J. Sant. ex Haf. \& Poelt

(2) Wolf \& Sipman 1877 [ECOSUR].

Caloplaca galactophylla (Tuck.) Zahlbr.

(13) saxícola sobre roca calcárea, Wolf \& Chanona 2665, 2669.

Candelaria fibrosa (Fr.) Müll. Arg.

Comitán, Meza (Dávalos de Guzmán et al. 1972: 15). — Breedlove 57422.

Canoparmelia amazonica (Nyl.) Elix \& Hale

Hale 19972 [DUKE, LD, US] (Hale 1976a: 16, as Pseudoparmelia).

Canoparmelia caroliniana (Nyl.) Elix \& Hale

Hale 19900, 20104, 20144, 20215, 20310, 20333 [US] (Hale 1976a: 24, as

Pseudoparmelia).

Canoparmelia cryptochlorophaea (Hale) Elix \& Hale

Lagunas de Colón, Mpio. Comitán, 15 40’N, 91 59’W, 620 m, bosque perturbado con el chicle, Sapotaceae y Ficus sp. como especies dominantes, epífita dentro de una copa de un árbol del dosel, 3 Mar. 1996, Wolf 2488; TLC: sekikaic, caperatic acid, atranorin. (13) epífita sobre rama de Bursera a 4.50 m, Wolf \& Chanona 2641; TLC: sekikaic, caperatic acid, atranorin.

Canoparmelia salacinifera (Hale) Elix \& Hale

Km 956 on Hwy. 190, west of Ocozocoautla, Hale 20607 [US] (Hale \& Kurokawa 1964:

157, as Parmelia; Hale 1976a: 46, as Pseudoparmelia).

Canoparmelia texana (Tuck.) Elix \& Hale

Hale 20278 [US] (Hale 1976a: 53, as Pseudoparmelia).

Catapyrenium pilosellum 0 . Breuss

(4) Wolf \& Sipman 2027.

Catinaria versicolor (Fée) Sipman $=$ Lopezaria versicolor.

Cetrariastrum cirrhatum (E. Fr.) W. Culb. \& C. Culb. = Everniastrum cirrhatum.

Cetrariastrum lipidiferum (Hale \& M. Wirth) W. Culb. \& C. Culb. = Everniastrum lipidiferum.

Cetrariastrum nigrociliatum (B. de Lesd.) W. Culb. \& C. Culb. = Everniastrum nigriciliatum.

Cetrariastrum vexans Zahlbr. ex W. Culb. \& C. Culb. = Everniastrum vexans. 
Cetrelia cetrarioides (Del. ex Duby) Culb. \& C. Culb. s.I.

(1) Wolf \& Sipman 1781.

Chaenotheca chrysocephala (Turn. ex Ach.) Th. Fr.

(5) Wolf \& Sipman 2088.

Chaenothecopsis rubina Tibell

Lagunas de Montebello, Kalb \& Plöbst s.n. (Tibell 1996: 47).

Chrysothrix candelaris (L.) Laundon

(3) Wolf \& Sipman 1982; (6) Wolf \& Sipman 2166 [ECOSUR]; Breedlove \& Bourell 67166, 68524.

Cladia aggregata (Sw.) Nyl.

Lagos de Monte Bello near the México-Guatemala border, c. 50 km southeast of Comitán, Hale 20466 [H]; above trail, above Parab, north of Mapastepec, 6 Nov. 1945, Sharp s.n. [COLO]; north of Mapastepec, 6 Nov. 1945, Sharp s.n. [US, hb. Evans]; trail over sierra from Pueblo Nuevo Solistahuacán to Simojovel, 24 dec. 1962, Sharp s.n. [WIS] (records from unpublished list of specimens, produced by Filson in 1981 in conjunction with his monograph of Cladia, p. 146). - (5) Wolf \& Sipman 2089.

Cladina confusa (R. Sant.) Follm. \& Ahti

Breedlove 68798 [CAS], det. T. Ahti 1992.

Cladina subtenuis (des Abb.) Hale \& W. Culb.

Breedlove 58369; Breedlove \& Bourell 67109, both det. T. Ahti 1995.

Cladonia botryocarpa G. K. Merrill

(12) Wolf 1698a, det. T. Ahti 1995.

Cladonia caribaea Stenroos

Lagunas de Montebello, 1450 m, Kalb \& Plöbst 14164 [hb. Kalb] (Stenroos 1989: 260). —

(2) Wolf \& Sipman 1905; (5) Wolf \& Sipman 2146, det. T. Ahti 1995.

Cladonia cartilaginea Müll. Arg.

Breedlove 67535 [CAS], det. T. Ahti 1992.

Cladonia ceratophylla (Sw.) Spreng.

(5) Wolf \& Sipman 2103, det. T. Ahti 1995; Breedlove 57528, det. T. Ahti 1995.

Cladonia chlorophaea (Flk. ex Sommerf.) Spreng.

Breedlove 57079 [CAS], det. T. Ahti 1992.

Cladonia corymbites Nyl.

Breedlove 58337, det. T. Ahti 1995.

Cladonia corymbosula Nyl.

Breedlove 67533, 69052 [CAS], det. T. Ahti 1992.

Cladonia dactylota Tuck.

(1) Wolf \& Sipman 1756; det. T. Ahti 1995.

Cladonia didyma (Fée) Vain. var. didyma

(2) Wolf \& Sipman 1869; (12) Wolf 1698; det. T. Ahti 1995; Breedlove 57090, det. T.

Ahti 1995; Breedlove 26851.

Cladonia dimorphoclada Robbins

Breedlove \& Bourell 67109a, det. T. Ahti 1995; new to Mexico!

Cladonia furcata (Hudson) Schrader

(2) Wolf \& Sipman 1865; det. T. Ahti 1995; Breedlove 51822, 55901.

Cladonia macilenta Hoffm.

Breedlove 68494 [CAS], det. T. Ahti 1992. 
Cladonia mexicana Vain.

Breedlove 68493 [CAS], det. T. Ahti 1992.

Cladonia ochrochlora Flk.

Breedlove 67244, 68238 [CAS], det. T. Ahti 1992.

Cladonia peziziformis (With.) Laund.

Breedlove 57078, teste T. Ahti 1995.

Cladonia pocillum (Ach.) O. Rich.

Breedlove 68297, 68302, 68303, 68305, 71385 [CAS], det. T. Ahti 1992.

Cladonia pyxidata (L.) Hoffm.

(4) Wolf \& Sipman 2030, det. T. Ahti 1995.

Cladonia ramulosa (With.) Laund. s.I.

Breedlove 67243 [CAS], det. T. Ahti 1992.

Cladonia scabriuscula (Del. in Duby) Nyl.

Breedlove 67818 [CAS], det. T. Ahti 1992.

Cladonia subpityrea Sandst. (Yoshimura \& Sharp 1968: 110) most probably a misidentification, as the species is now considered to be not native to the Neotropics; perhaps confused with $C$. dactylota, acc. to T. Ahti (in litt.).

Cladonia squamosa (Scop.) Hoffm. var. squamosa

(2) Wolf \& Sipman 1869a; (3) Wolf \& Sipman 1984; (10) Wolf 1725 ; det. T. Ahti 1995;

Breedlove 58193.

Cladonia subradiata (Vain.) Sandst.

Breedlove 57528, among Cladonia ceratophylla, det. T. Ahti 1995.

Cladonia subsquamosa Kremp.

Breedlove 57079.

Coccocarpia erythroxyli (Spreng.) Swinsc. \& Krog

(5) Wolf \& Sipman 2155; Breedlove 71353.

Coccocarpia palmicola (Spreng.) Arvidss. \& Gallow.

(2) Wolf \& Sipman 1898; (5) Wolf \& Sipman 2084.

Coccocarpia pellita (Ach.) Müll. Arg.

Breedlove \& Bourell 67856.

Coenogonium linkii Ehrenb.

(5) Wolf \& Sipman 2110, 2132.

Coenogonium moniliforme Tuck.

Breedlove \& Bourell 67678.

Collema furfuraceum (Arn.) DR. var. furfuraceum

50 km W of Tuxtla Gutiérrez, Hwy 190, trees, decid. mist forest, 1040 m, Hale 19946 [US] (Degelius 1974: 181).

Collema furfuraceum var. luzonense (Räs.) Degel.

Road to El Suspiro, 5-7 km N of Berriozábal, decid. forest, mist forest, 920 m, Hale 20094 [US] (Degelius 1974: 181).

Collema glaucophthalmum Nyl. var. glaucophthalmum (2) Wolf \& Sipman 1805; (3) Wolf \& Sipman 2003.

Collema glaucophthalmum Nyl. var. implicatum (Nyl.) Degel.

Pueblo Nuevo Solistahuacán: trail to Yerba Buena Mission cloud forest preserve, trunk of low shrubby tree, altitude 1900-2000 m, 1963, S.S. Tillet 636-24 [US] (Degelius 1974: 169). 
Collema leptaleum Tuck. var. leptaleum

Road to El Suspiro, 5-7 km N of Berriozábal, scrubby decid. pasture, mist forest, decid. trees, 920 m, Hale 20103 [US] (Degelius 1974: 108).

Cryptothecia rubrocincta (Ehrenb.) Thor

(5) Wolf \& Sipman 2072, 2128; Breedlove 58371.

Dermatocarpon miniatum (L.) Mann

(4) Wolf \& Sipman 2021.

Dibaeis absolutus (Tuck.) Kalb \& Gierl

NE of Cerro Boquerón, 2300 m, 1988, Breedlove 68857 (Gierl \& Kalb 1993: 614).

Dictyonema glabratum (Spreng.) D. Hawksw.

(2) Wolf \& Sipman 1851; Breedlove 58349.

Dictyonema sericeum (Sw.) Berk.

(2) Wolf \& Sipman 1888.

Diploschistes cinereocaesius (Sw. ex Ach.) Vain.

Breedlove 57442a, Breedlove \& Bourell 67330.

Dirinaria applanata (Fée) Awas.

Oaxaca-Chiapas, on stones, Hale 19892 [US] (Awasthi 1975: 83).

Dirinaria confluens (Fr.) Awas.

Zapota, Hale 19978 [US] (Awasthi 1975: 31).

Erioderma mollissimum (Samp.) DR.

(2) Wolf \& Sipman 1811, 1897; (3) Wolf \& Sipman 1998 [ECOSUR].

Everniastrum cirrhatum (E. Fr.) Hale ex Sipman

6 km NW of Rincón Chamula, Culberson 16523 [DUKE] (Culberson \& Culberson 1981:

285, as Cetrariastrum). - Breedlove 58237.

Everniastrum lipidiferum (Hale \& M. Wirth) Hale ex Sipman

El Sumidero, Hale 20064 [US holotype, DUKE] (Culberson \& Culberson 1981: 287, as Cetrariastrum); $2 \mathrm{~km} \mathrm{~N}$ highway 190 on road to Puebla Nueva, Hale 20172 [US] (Hale \& Wirth 1971: 37, as Parmelia; Culberson \& Culberson 1981: 287, as Cetrariastrum). Breedlove 68576; (12) Wolf 1692.

Everniastrum neocirrhatum (Hale \& M. Wirth) Hale ex Sipman

Km 1145 on highway 190, W of San Cristóbal, Hale 20225 [US] (Hale \& Wirth 1971: 38, as Parmelia); 14 km W of San Cristóbal, Hale 20579 [US] (Hale \& Wirth 1971: 38, as Parmelia); S of Teopisca, Hale 20524 [US] (Hale \& Wirth 1971: 38, as Parmelia).

Everniastrum nigrociliatum (B. de Lesd.) Hale ex Sipman

7 km W of San Cristóbal, Culberson 16507 [DUKE] (Culberson \& Culberson 1981: 291, as Cetrariastrum). - Breedlove 69895; (2) Wolf \& Sipman 1892 (cf.); (3) Wolf \& Sipman 2013.

Everniastrum vexans (Zahlbr. ex W. Culb. \& C. Culb.) Hale ex Sipman

$6 \mathrm{~km}$ NW of Rincón Chamula, Culberson 16520 [DUKE] (Culberson \& Culberson 1981: 296, as Cetrariastrum). - (1) Wolf \& Sipman 1737, 1778; (2) Wolf \& Sipman 1818, 1862 , 1894; (5) Wolf \& Sipman 2049, 2079; Breedlove 58097b, 58163, 65658; Breedlove \& Bourell 67172, 67263, 67263B, 67338, 68146, 68312, 68416; Breedlove 68822,e 68972.

Flavoparmelia caperata (L. ) Hale

(1) Wolf \& Sipman 1772; (2) Wolf \& Sipman 1840, 1868, 1870; Breedlove \& Bourell 67333, 68321. 
Flavopunctelia flaventior (Stirt.) Hale

(1) Wolf \& Sipman 1744; (2) Wolf \& Sipman 1875; Breedlove \& Bourell 67713; Breedlove 57416.

Flavopunctelia praesignis (Nyl.) Hale

(2) Wolf \& Sipman 1896; Breedlove \& Bourell 68154; Breedlove 69157.

Fuscopannaria leucosticta P.M. Jørg.

(3) Wolf \& Sipman 2005

Glyphis cicatricosa Ach.

(7) Wolf \& Sipman 2195 [ECOSUR], 2251.

Graphina acharii (Fée) Müll. Arg.

El Suspiro, Hale 20230, 20237 [US] (Wirth \& Hale 1963: 72); north of Tuxtla Gutiérrez,

Hale 20076 [US] (Wirth \& Hale 1963: 72); Lagos de Monte Bello, Hale 20437 [US] (Wirth \& Hale 1963: 72).

Graphina confluens (Fée) Müll. Arg.

El Suspiro, Hale 20155, 20169 [US] (Wirth \& Hale 1963: 78). — (7) Wolf \& Sipman 2196, 2252, 2258.

Graphina elongata (Vain.) Zahlbr.

Lagos de Monte Bello, Hale 21069 [US] (Wirth \& Hale 1963: 74).

Graphina insignis (Vain.) Zahlbr.

Lagos de Monte Bello, Hale 20499 [DUKE, REN, S, TNS, US] (Wirth \& Hale 1963: 74).

Graphina macella (Kremp.) Müll. Arg.

Southeast of San Cristóbal, Hale 20275 [US] (Wirth \& Hale 1963: 82). - (3) Wolf \& Sipman 1926.

Graphina sulcata Fink

El Suspiro, Hale 20233, 20086 [US] (Wirth \& Hale 1963: 88).

Graphina virginalis (Nyl.) Müll. Arg.

El Suspiro, Hale 20225 [US] (Wirth \& Hale 1963: 90).

Graphina virginea (Eschw.) Müll. Arg.

(7) Wolf \& Sipman 2197, 2262; chemistry OK, but no spores found.

Graphis afzelii Ach.

Road to El Suspiro, Hale 20099 [S, TNS, US] (Wirth \& Hale 1963: 91).

Graphis caesiella Vain.

(6) Wolf \& Sipman 2165 [ECOSUR], (7) Wolf \& Sipman 2285?

Graphis flexibilis Kremp.

West of Tuxtla Gutiérrez, Hale 19894 [US] (Wirth \& Hale 1963: 94).

Graphis glaucescens Fée

(7) Wolf \& Sipman 2243

Graphis glaucopsis Wirth \& Hale

El Suspiro, Hale 20219 [US holotype, S, TNS] (Wirth \& Hale 1963: 95).

Graphis grammatica Nyl.

El Sumidero, Tuxtla Gutiérrez, Hale 20042 [US] (Wirth \& Hale 1963: 96).

Graphis grammitis Fée

(5) Wolf \& Sipman 2153.

Graphis longula Kremp.

North of Berriozábal, Hale 20225 [US] (Wirth \& Hale 1963: 98). 
Graphis stygioarachnoidea Wirth \& Hale

Lagos de Monte Bello, Hale 20399 [US holotype] (Wirth \& Hale 1963: 99).

Graphis triticea Nyl.

(2) Wolf \& Sipman 1835; (3) Wolf \& Sipman 1938.

Gyrostomum scyphuliferum (Ach.) Nyl.

(13) epífita sobre rama de Bursera a $4.0 \mathrm{~m}$, Wolf \& Chanona 2648.

Haematomma flexuosum Hillm.

Breedlove \& Bourell 68255a (Staiger \& Kalb 1995: 112).

Haematomma rufidulum (Fée) Massal.

(1) Wolf \& Sipman 1749.

Helminthocarpon leprevostii Fée

(13) epífita sobre tronco de Ceiba, colectada a $1.50 \mathrm{~m}$, Wolf \& Chanona 2646.

Heterodermia albicans (Pers.) Swinsc. \& Krog

El Zapotal, Tuxtla Gutiérrez, 920 m, Hale 19974 [US, hb. Kurokawa] (Kurokawa 1962:

35, as Anaptychia domingensis). - (4) Wolf \& Sipman 2024; (6) Wolf \& Sipman 2171,

2172 [ECOSUR]; (7) Wolf \& Sipman 2213a cf.; Breedlove \& Bourrell 67682b.

Heterodermia antillarum (Vain.) Swinsc. \& Krog $50 \mathrm{~km} \mathrm{~W}$ of Tuxtla Gutiérrez on highway

190, 1040 m, Hale 19931 [US] (Kurokawa 1962: 37, as Anaptychia tropica var. antillarum). - Breedlove \& Bourell 68118.

Heterodermia barbifera (Nyl.) K. P. Singh

Lagos de Monte Bello, 1220 m, Hale 20405 [US] (Kurokawa 1962: 99, as Anaptychia). -

(2) Wolf \& Sipman 1806 [ECOSUR]; (5) Wolf \& Sipman 2050.

Heterodermia casarettiana (Massal.) Trevis.

El Suspiro, $10 \mathrm{~km} \mathrm{~N}$ of Berriozábal, Hale 20228A [US] (Kurokawa 1962: 57, as

Anaptychia; Kurokawa 1973: 601, map, as Anaptychia). - (1) Wolf \& Sipman 1768; (3)

Wolf \& Sipman 1950; (5) Wolf \& Sipman 2080; Breedlove \& Bourell 67147, 67482, 67524,

67542, 67574, 67641, 67682; Breedlove 71150.

Heterodermia circinnalis (Zahlbr.) W. A. Weber

Breedlove \& Bourell 67337.

Heterodermia comosa (Eschw.) Follm. \& Redon

El Zapotal, Tuxtla Gutiérrez, 920 m, Hale 19995 [US, Kurokawa] (Kurokawa 1962: 104, as Anaptychia). - Breedlove \& Bourell 67385A.

Heterodermia crocea R. C. Harris

Lagos de Monte Bello, Hale 20390 [US] (Harris 1990: 78). - Breedlove \& Bourell

67542a; (5) Wolf \& Sipman 2082. The Hale specimen was recorded by Kurokawa (1962:

63) as Anaptychia corallophora.

Heterodermia dissecta (Kurok.) Awasthi

El Sumidero, near Tuxtla Gutiérrez, Hale 20017 [US] (Kurokawa 1962: 39, as Anaptychia var. koyana). The status of the variety was not confirmed in recent publications, therefore the required recombination into Heterodermia is not made here, and the material is provisionally attributed to the main variety.

Heterodermia echinata (Tayl.) W. Culb.

Breedlove 57418.

Heterodermia flabellata (Fée) Awasthi var. flabellata

El Sumidero Canyon, 1220 m, Hale 20047 [US] (Kurokawa 1962: 53, as Anaptychia). 
Heterodermia galactophylla (Tuck.) Trev.

(2) Wolf \& Sipman 1807.

Heterodermia granulifera (Ach.) W. Culb.

$2 \mathrm{~km} \mathrm{~N}$ of highway 190, road to Puebla Nueva, 1070 m, Hale 20178 [US] (Kurokawa 1962: 37, as Anaptychia).

Heterodermia isidiophora (Nyl.) Awasthi

Lagos de Monte Bello, 50 km SE of Comitán, 1220 m, Hale 20364 [US] (Kurokawa 1962: 34, as Anaptychia).

Heterodermia lamelligera (Tayl.) Follm. \& Redon

(1) Wolf \& Sipman 1753 [ECOSUR]; (2) Wolf \& Sipman 1895; (3) Wolf \& Sipman 1976;

(10) Wolf 1729; (11) Wolf 1673; Breedlove 57103. The yellow pigmentation of the lower side is very variable, ranging from abundant to absent.

Heterodermia leucomelos (L.) Poelt ssp. boryi (Fée) Swinsc. \& Krog

(2) Wolf \& Sipman 1808 [ECOSUR]; (5) Wolf \& Sipman 2131 [ECOSUR]; Breedlove \& Bourell 67385, 67465, 67506, 67569; Breedlove 68788.

Heterodermia leucomelos (L.) Poelt ssp. leucomelos

El Zapotal, Tuxtla Gutiérrez, 920 m, Hale 19994 [US, hb. Kurokawa] (Kurokawa 1962:

76, as Anaptychia leucomelaena fo. albociliata); El Sumidero, Tuxtla Gutiérrez, 1220 m, Hale 20010 [US], 20015 [US, hb. Kurokawa] (Kurokawa 1962: 76, as Anaptychia leucomelaena fo. leucomelaena); $2 \mathrm{~km} \mathrm{~N}$ of highway 190 on road to Puebla Nueva, $1070 \mathrm{~m}$, Hale 20260 [US] (Kurokawa 1962: 77, as Anaptychia leucomelaena fo. palmiformis). Breedlove 67169, Breedlove \& Bourell 67702, 67836, 67851, 68322, 68442; (1) Wolf \& Sipman 1786.

Heterodermia lutescens (Kurok.) Follm.

Monte Ovando, 25 Mar 1932, Matuda 46 [hb. Asahina holotype, hb. Kurokawa] (Kurokawa 1961: 56, 1962: 79, as Anaptychia; Kurokawa 1973: 590, map, as Anaptychia); Lagos de Monte Bello near the Mexico-Guatemala border, on deciduous trees, 1220 m, Hale 20443 [US] (Kurokawa 1961: 56, as Anaptychia). - Breedlove \& Bourell 67169a; Breedlove 57016, 58097.

Heterodermia magellanica (Zahlbr.) Swinsc. \& Krog

18 km SE of San Cristóbal on highway 190, 2340 m, Hale 20257, 20533 [US] (Kurokawa 1962: 67, 68, as Anaptychia magellanica var. pectinata fo. pectinata). The status of var. and fo. pectinata has not been confirmed in recent publications; therefore the required new combination into Heterodermia is not made here and the material is provisionally included in the main division of the species.

Heterodermia obscurata (Nyl.) Trevis.

50 km W of Tuxtla Gutiérrez on highway 190, 1040 m, Hale 19940 [US, hb. Kurokawa] (Kurokawa 1962: 51, as Anaptychia). - Breedlove \& Bourell 67215, 67574a.

Heterodermia podocarpa (Bél.) Awasthi

Breedlove 58100A [CAS], det. H. Sipman 1988.

Heterodermia polyrhiza (Kurok.) Kurok.

About 40 km SE of Comitán, San Lorenzo Ranch, 1220 m, March 25, 1960, Hale 20478 [TNS holotype, US] (Kurokawa 1962: 33, as Anaptychia). — Breedlove \& Bourell 68114, 68115.

Heterodermia speciosa (Wulf.) Trevis.

$2 \mathrm{~km} \mathrm{~N}$ of highway 190 on road to Puebla Nueva, 1070 m, Hale 20250a [US] (Kurokawa 
1962: 27, as Anaptychia pseudospeciosa var. tremulans); Lagos de Monte Bello, $1220 \mathrm{~m}$, Hale 20430 [US] (Kurokawa 1962: 27, as Anaptychia pseudospeciosa var. tremulans). Breedlove \& Bourell 67378.

Heterodermia squamulosa (Degel.) W. Culb.

18 km SE of San Cristóbal, Hale 20279 [US] (Kurokawa 1962: 60, as Anaptychia). (1) Wolf \& Sipman 1751; (12) Wolf 1694. Breedlove \& Bourell 67182, 67210, 67657, 68151, 68153; Breedlove 57091, 58303, 68758A.

Heterodermia subcomosa (Nyl.) Elix

Breedlove 69713 [CAS], det. H. Sipman 1989.

Heterodermia subisidiosa (Kurok.) Kurok.

$8 \mathrm{~km}$ E of Teopisca, Hale 20354 [TNS holotype, US] (Kurokawa 1962: 68, as Anaptychia magellanica var. pectinata fo. subisidiosa Kurok.; Kurokawa 1973, as Anaptychia subisidiosa).

Heterodermia tropica (Kurok.) Sipman

50 km W of Tuxtla Gutiérrez, Hale 19916 [US] (Kurokawa 1962: 36, as Anaptychia tropica var. tropica). - (2) Wolf \& Sipman 1810 [ECOSUR]; Breedlove \& Bourell 67676A, 67682d, 67706, 68092, 68156; Breedlove 57421, 57423, 57439. The nomenclatural combination, published by Marcano et al. (1996: 206), was overlooked in a recent survey of the genus (Kurokawa 1998).

Heterodermia verrucifera (Kurok.) W.A. Weber

14 km W of San Cristóbal, 2340 m, Hale 20554 [US] (Kurokawa 1962: 77, as Anaptychia leucomelaena fo. verrucifera).

Hyperphyscia syncolla (Tuck.) Kalb

Breedlove 57417 [CAS]

Hypotrachyna bogotensis (Vain.) Hale

Hale 20266, 20541, 20542 [US] (Hale 1975: 24). - (2) Wolf \& Sipman 1867, 1889;

Breedlove \& Bourell 67211, 68149; Breedlove 58161b.

Hypotrachyna consimilis (Vain.) Hale

Hale 20375 [US] (Hale 1975: 29). - (5) Wolf \& Sipman 2126 [ECOSUR].

Hypotrachyna costaricensis (Nyl.) Hale

Hale 20036, 20046, 20479 [US] (Hale 1975: 30). - (5) Wolf \& Sipman 2051, 2112;

Breedlove \& Bourell 67214a.

Hypotrachyna croceopustulata (Kurok.) Hale

(3) Wolf \& Sipman 1985.

Hypotrachyna dactylifera (Vain.) Hale

Hale 20288, 20375 [US] (Hale 1975: 31). - (4) Wolf \& Sipman 2035; Breedlove \& Bourell

68167a.

Hypotrachyna densirrhizinata (Kurok.) Hale

(2) Wolf \& Sipman 1872; (3) Wolf \& Sipman 1971.

Hypotrachyna endochlora (Leight.) Hale

Breedlove \& Bourell 67357.

Hypotrachyna exsplendens (Hale) Hale

Virgin upland jungle, El Suspiro, Hale 20229 [US] (Hale \& Kurokawa 1964: 178, as

Parmelia). - Breedlove \& Bourell 68152, 68462.

Hypotrachyna formosana (Zahlbr.) Hale $=H$. osseoalba. 
Hypotrachyna gondylophora (Hale) Hale

Hale 19920 [US] (Hale 1975: 40). - Breedlove 68802.

Hypotrachyna imbricatula (Zahlbr.) Hale

Hale 20021, 20197, 20365, 20455, 21202 [US] (Hale 1975: 41). - (5) Wolf \& Sipman

2066; Breedlove \& Bourell 67477, 67827, 68463; Breedlove 57426.

Hypotrachyna isidiocera (Nyl.) Hale

Hale 20219 [DUKE, US] (Hale 1975: 44). - (1) Wolf \& Sipman 1735.

Hypotrachyna laevigata (Smith) Hale

Hale 19885 [US] (Hale 1975: 45). - Breedlove \& Bourell 68496.

Hypotrachyna microblasta (Vain.) Hale

Hale 20038, 20210, 20221, 20353, 20385, 20553 [US] (Hale 1975: 48). — Breedlove \& Almeda 57429 .

Hypotrachyna osseoalba (Vain.) Park \& Hale

Hale 19942, 20110, 20417, 20469, 20806 [US] (Hale 1975: 38, as Hypotrachyna formosana). - (4) Wolf \& Sipman 2034; Breedlove 58366a.

Hypotrachyna physcioides (Nyl.) Hale

Breedlove \& Bourell 68415.

Hypotrachyna prolongata (Kurok.) Hale

18 km southeast of San Cristóbal, Hale 20286 [US] (Hale \& Kurokawa 1964: 183, as Parmelia).

Hypotrachyna pulvinata (Fée) Hale

Hale 20330, 20571 [US] (Hale 1975: 59). — (1) Wolf \& Sipman 1784; (2) Wolf \& Sipman 1886; (10) Wolf 1722; Breedlove \& Bourell 67274; Breedlove 58161.

Hypotrachyna reducens (Nyl.) Hale

Breedlove 67289A [CAS], det. H. Sipman 1989.

Hypotrachyna rockii (Zahlbr.) Hale

Hale 20214, 20242, 20290, 20534, 20561 [US] (Hale 1975: 62). - (2) Wolf \& Sipman 1906; (11) Wolf 1682; Breedlove \& Bourell 67357a, 68167; Breedlove 51830, 57426a;

(1) Wolf \& Sipman 1773.

Hypotrachyna subaffinis (Zahlbr.) Hale

Hale 20352, 20384 [US] (Hale 1975: 66).

Imshaugia sp. c. is.

(3) Wolf \& Sipman 1992. TLC: thamnolic acid; the specimen belongs to the isidiate morph

of "Parmeliopsis" angustior (Nyl.) Nyl.; this morph occurs also in Costa Rica and Colombia.

Lecanactis epileuca (Nyl.) Tehler

(5) Wolf \& Sipman 2058a [ECOSUR]; (7) Wolf \& Sipman 2248.

Lecanora caesiorubella Ach. ssp. glaucomodes (Nyl.) Imsh. \& Brodo

Km. 956 on highway 190, west of Ocozocoautla, 790 m, Hale 20599 [US]; 2 km N of highway 190 on road to Puebla Nueva, W of Chiapas, 1070 m, Hale 20181 [US] (Imshaug \& Brodo 1966: 19); ca. 25 km NW of Comitán de Domínguez, on deciduous trees in shady, dry Quercus-Pinus forest, 2150 m, Kalb \& Plöbst s.n., in: K. Kalb, Lichenes Neotropici 98 [B] (Kalb 1982: 7). — Breedlove 57433; (1) Wolf \& Sipman 1767.

Lecanora oreinoides (Koerb.) Hertel \& Rambold

Breedlove \& Bourell 67124. 
Leptogium adpressum Nyl.

(1) Wolf \& Sipman 1771.

Leptogium azureum (Sw.) Mont.

Carretera Panamericana entre San Juan y San Cristóbal de las Casas, A. Loeza 643

[IBUG] (Guzmán-Dávalos \& Alvarez 1987: 222. - (1) Wolf \& Sipman 1766; (2) Wolf

\& Sipman 1860; (3) Wolf \& Sipman 1910a; (5) Wolf \& Sipman 2109; Breedlove 57161, 57415, 58174, 58176.

Leptogium burgessii (L.) Mont.

(1) Wolf \& Sipman 1736; (2) Wolf \& Sipman 1829, 1834 [ECOSUR]; Breedlove 58178. Leptogium cochleatum (Dicks.) P. M. Jørg. \& P. W. James

(1) Wolf \& Sipman 1779; (2) Wolf \& Sipman 1859 [ECOSUR]; (3) Wolf \& Sipman 1910;

(7) Wolf \& Sipman 2254a; Breedlove 57157, 57425, 57431, 58130.

Leptogium coralloideum (Mey. \& Flot.) Vain.

Breedlove 57084, 57437.

Leptogium hibernicum Mitch. ex P. M. Jörg.

Breedlove 57444.

Leptogium hypotrachynum Müll. Arg.

Breedlove 57014, 57431b.

Leptogium marginellum (Sw.) S. F. Gray

Breedlove 57015.

Leptogium olivaceum (Hook.) Zahlbr.

Breedlove 51825, 58245; (2) Wolf \& Sipman 1817.

Leptogium phyllocarpum (Pers.) Nyl.

(2) Wolf \& Sipman 1804.

Leptogium vesiculosum (Sw.) Malme

Breedlove 53209; (2) Wolf \& Sipman 1809.

Lobaria amplissima (Hoffm.) Schreb.

North of Berriozábal, Hale 20090 [US] (Hale 1961).

Lobaria crenulata (Hook. \& Kunth) Trev.

Breedlove 57434; (1) Wolf \& Sipman 1780, 1782.

Lobaria dissecta (Sw.) Räusch.

Breedlove \& Bourell 67869; (3) Wolf \& Sipman 2008.

Lobaria pallida (Hook. in Kunth) Trev.

(3) Wolf \& Sipman 2011; (10) Wolf 1726.

Lobaria peltigera (Del.) Vain. s. lat.

Breedlove \& Almeda 57574.

Lobaria pulmonaria (L.) Hoffm.

(3) Wolf \& Sipman 1935, 2007; (10) Wolf 1709; Breedlove 51823.

Lobaria subexornata Yoshim.

(1) Wolf \& Sipman 1745; (10) Wolf 1721.

Lopezaria versicolor (Fée) Kalb \& Haf.

El Suspiro, 10 km N of Berriozábal, Hale 20240 [US] (Sipman 1983: 230, as Catinaria versicolor); $18 \mathrm{~km}$ SE of San Cristóbal, Hale 20273 [US] (Sipman 1983: 231, as Catinaria versicolor ). - (1) Wolf \& Sipman 1743, 1789; (3) Wolf \& Sipman 1912, 1927, 1947 [ECOSUR], 1948; (5) Wolf \& Sipman 2085, 2290; Breedlove \& Bourell 67206, 67638, 67759a; Breedlove 57430. 
Maronina multifera (Nyl.) Haf. \& Rogers

(13) epífita sobre tronco de Ficus, colectada a $1.0 \mathrm{~m}$, Wolf \& Chanona 2601.

Mazosia ocellata (Nyl.) R. C. Harris

(5) Wolf \& Sipman 2055.

Mazosia phyllosema (Nyl.) Zahlbr.

Urwald bei den Palenque Ruinen, 150 m, Kalb [hb. Kalb] (Kalb \& Vezda 1988: 203).

Mazosia rotula (Mont.) Massal.

Feuchter Urwald bei den Ruinen von Palenque, 150 m, Kalb [hb. Kalb] (Kalb \& Vezda 1988: 209).

Megalospora sulphurata Mey. \& Flot. var. nigricans (Müll. Arg.) Riddle

(2) Wolf \& Sipman 1841, 1899; (3) Wolf \& Sipman 1958 [ECOSUR]; (5) Wolf \& Sipman

2151 [ECOSUR]; Breedlove \& Bourell 67759.

Megalospora tuberculosa (Fée) Sipman

El Suspiro N of Berriozábal, Hale 20232 [US] (Sipman 1983: 231). - (3) Wolf \& Sipman 1943, 1949, 1965; (5) Wolf \& Sipman 2087.

Megalospora vs. tuberculosa (Fée) Sipman

Breedlove 67221. A completely sorediate plant without apothecia, perhaps deserving an independent taxonomic status and conspecific with "Bombyliospora incana"?

Mycocalicium albonigrum (Nyl.) Tibell

10 km NW of Comitán de Domínguez, Kalb \& Plöbst s.n. (Tibell 1996: 49).

Mycocalicium americanum (R. Sant.) Tibell

Close to Jitotol, on Hwy. 195, Kalb \& Plöbst s.n.; 25 km NW of Comitán de Domínguez,

Kalb \& Plöbst S207 (Tibell 1996: 50).

Mycocalicium subtile (Pers.) Szatala

Lagunas de Montebello, Kalb \& Plöbst s.n. (Tibell 1996: 52).

Myriotrema bahianum (Ach.) Hale

(5) Wolf \& Sipman 2073.

Myriotrema glaucescens (Nyl.) Hale

(7) Wolf \& Sipman 2207, 2245.

Myriotrema hartii (Müll. Arg.) Hale

Breeedlove \& Bourell 67822. Without apothecia; from 1600 m, Montebello.

Myriotrema neofrondosum Sipman?

Breedlove \& Bourell 67640A. From 1500 m alt., Munic. Angel Albino Corzo. Chemistry needs to be redone.

Myriotrema wightii (Tayl.) Hale

(6) Wolf \& Sipman 2169.

Nadvornikia hawaiensis (Tuck.) Tibell

22 km N of Tuxtla Gutiérrez, El Sumidero Cañón, Kalb \& Plöbst s.n. (Tibell 1996: 56).

Normandina pulchella (Borr.) Nyl.

(2) Wolf \& Sipman 1815.

Ocellularia cavata (Ach.) Müll. Arg.

(5) Wolf \& Sipman 2099, 2106.

Ocellularia comparabilis (Kremp.) Müll. Arg.

(7) Wolf \& Sipman 2242.

Ocellularia domingensis (Fée in Nyl.) Müll. Arg.

(5) Wolf \& Sipman 2071, 2100 [ECOSUR], 2118, 2123, 2291. 
Ocellularia perforata (Leight.) Müll. Arg.

(5) Wolf \& Sipman 2070.

Ocellularia cf. perforata (Leight.) Müll. Arg.

(6) Wolf \& Sipman 2158, 2173; (7) Wolf \& Sipman 2209, 2211, 2223, 2246, 2260, 2286, 2287. These specimens agree by their chemistry and ascocarp anatomy, but differ by their thicker thalli of a more olivaceous colour. They seem also unusual because of the xeric habitat.

Ocellularia tenuis (Hale) Hale

(5) Wolf \& Sipman 2129.

Ochrolechia africana Vain.

(13) epífita sobre rama de Ficus, colectada a $2.5 \mathrm{~m}$, Wolf \& Chanona 2600.

Ochrolechia isidiata (Malme) Vers.

Ixtapa/Bochil, Kalb \& Plöbst 10875 [hb. Kalb] (Brodo 1991: 749).

Ochrolechia mexicana Vain.

Comitán de Domínguez, Kalb \& Plöbst 17853 [hb. Kalb] (Brodo 1991: 752).

Oropogon caespitosus Essl.

(2) Wolf \& Sipman 1816, 1890 [ECOSUR]; (3) Wolf \& Sipman 1972.

Oropogon formosanus Asah.

(10) Wolf 1709a.

Oropogon granulosus Essl.

(2) Wolf \& Sipman 1885.

Oropogon loxensis (Fée) Th. Fr.

(2) Wolf \& Sipman 1891; Breedlove 58202.

Oropogon mexicanus Essl.

(3) Wolf \& Sipman 1996; Breedlove 58158, 59898, 68878.

Pannaria conoplea (Ach.) Bory

(2) Wolf \& Sipman 1873.

Pannaria rubiginosa (Ach.) Bory

(2) Wolf \& Sipman 1842; Breedlove \& Bourell 67213.

Pannaria tavaresii P. M. Jørg.

(5) Wolf \& Sipman 2093a.

Parmelia aberrans (Vain.) Des Abbayes = Parmotrema xanthinum.

Parmelia abessinica Kremp. = Parmotrema abessinicum.

Parmelia argentina Kremp. = Parmotrema argentinum .

Parmelia austrosinensis Zahlbr. = Parmotrema austrosinense.

Parmelia chiapensis Hale = Parmotrema chiapense (Hale) Hale.

Parmelia conformata Vain. = Parmotrema conformatum.

Parmelia crinita Ach. = Parmotrema crinitum.

Parmelia cristifera Tayl. = Parmotrema cristiferum.

Parmelia dilatata Vain. = Parmotrema dilatatum.

Parmelia disparilis Zahlbr. = Parmotrema disparile.

Parmelia dominicana Vain. = Parmotrema dominicanum.

Parmelia ebulliens Hale = Parmotrema mesogenes (Nyl.) Hale.

Parmelia endosulphurea (Hillm.) Hale = Parmotrema endosulphureum.

Parmelia exsplendens Hale = Hypotrachyna exsplendens. 
Parmelia hababiana Gyeln. = Parmotrema hababianum.

Parmelia latissima Fée = Parmotrema latissimum.

Parmelia leucosemotheta Hue = Parmotrema leucosemothetum.

Parmelia lipidifera Hale \& M. Wirth = Everniastrum lipidiferum.

Parmelia madagascariacea (Hue) des Abb. = Parmotrema xanthinum.

Parmelia mellissii Dodge = Parmotrema mellissii.

Parmelia mesogenes Nyl. = Parmotrema mesogenes.

Parmelia miranda Hale = Parmotrema mirandum.

Parmelia mordenii Hale = Parmotrema mordenii.

Parmelia myelochroa Hale = Parmotrema myelochroum.

Parmelia neocirrhata Hale \& M. Wirth = Everniastrum neocirrhatum.

Parmelia peralbida Hale = Parmotrema peralbidum.

Parmelia praesorediosa Nyl. = Parmotrema praesorediosum .

Parmelia prolongata Kurok. = Hypotrachyna prolongata .

Parmelia rampoddensis Nyl. = Parmotrema rampoddense.

Parmelia reparata Stirton $=$ Rimeliella subcaperata.

Parmelia rudecta Ach. $=$ Punctelia rudecta.

Parmelia salacinifera Hale = Canoparmelia salacinifera.

Parmelia simulans Hale = Rimelia simulans.

Parmelia stuppea Tayl. = Parmotrema stuppeum.

Parmelia subarnoldii des Abb. = Parmotrema subarnoldii.

Parmelia subcrinita auct., non Nyl. = Parmotrema ultralucens.

Parmelia subrugata Kremp. = Parmotrema subrugatum.

Parmelia subsumpta Nyl. = Rimeliella subsumpta.

Parmelia subtinctoria Zahlbr. = Rimeliella subtinctoria.

Parmelia tinctorum Nyl. = Parmotrema tinctorum.

Parmelia ventricosa Hale \& Kurok. = Bulbothrix ventricosa.

Parmelia viridiflava Hale = Parmotrema viridiflavum.

Parmelia xanthina (Müll. Arg.) Vain. = Parmotrema xanthinum.

Parmelia zollingeri Hepp = Parmotrema zollingeri .

Parmeliella pannosa (Sw.) Müll. Arg.

(5) Wolf \& Sipman 2053, 2093 (B, ECOSUR); Breedlove 68623. The identification of

Nr. 2093 is uncertain, because the specimen is without ascocarps.

Parmelina antillensis (Nyl.) Hale = Parmotremopsis antillensis.

Parmelina dissecta (Nyl.) Hale = Parmelinopsis minarum.

Parmelina horrescens (Tayl.) Hale $=$ Parmelinopsis horrescens.

Parmelina phlyctina (Hale) Hale = Parmotremopsis phlyctina.

Parmelina spumosa (Asah.) Hale = Parmelinopsis spumosa.

Parmelinopsis horrescens (Tayl.) Elix \& Hale

Hale 20204, 20223, 20400a, 20414, 20549, 21086 [US] (Hale 1976b: 32, as Parmelina). -

(5) Wolf \& Sipman 2125.

Parmelinopsis minarum (Vain.) Elix \& Hale

Hale 20304 [US] (Hale 1976b: 27, as Parmelina dissecta).

Parmelinopsis spumosa (Asah.) Elix \& Hale

Hale 19897, 20110a, 20150 [US] (Hale 1976b: 46, as Parmelina). 
Parmotrema abessinicum (Kremp.) Hale

El Zapotal, Tuxtla Gutiérrez, Hale 19990 [US] (Hale 1965: 321, as Parmelia; cryptochlorophaeic acid-strain).

Parmotrema argentinum (Kremp.) Hale

El Zapotal, Tuxtla Gutiérrez, Hale 19991, 20005 [US] (Hale 1965: 322, as Parmelia);

El Sumidero, near Tuxtla Gutiérrez, Hale 20061 [US] (Hale 1965: 322, as Parmelia).

Parmotrema arnoldii (DR) Hale

Breedlove \& Bourell 68154A, 68155; (2) Wolf \& Sipman 1666.

Parmotrema austrosinense (Zahlbr.) Hale

South of Teopisca, Hale 20508 [US] (Hale 1965: 239, as Parmelia).

Parmotrema chiapense (Hale) Hale

Monte Ovando, I Jan 1925, Matuda 105 [TNS holotype, US] (Hale 1965: 323, as

Parmelia); Mt. Ovando, dec 1937, Matuda s.n. [MICH] (Hale 1965: 323, as Parmelia);

Mt. Ovando, Matuda 45 [TNS] (Hale 1965: 323, as Parmelia).

Parmotrema conformatum (Vain.) Hale

18 km SE of San Cristóbal, Hale 20296 [US] (Hale 1965: 271, as Parmelia); 50 km west of Tuxtla Gutiérrez, Hale 19930 [US] (Hale 1965: 271, as Parmelia); 40 km southeast of Comitán, Hale 20477 [US] (Hale 1965: 271, as Parmelia).

Parmotrema crinitum (Ach.) M. Choisy

50 km west of Tuxtla Gutiérrez on highway 190, Hale 19907 [DUKE, S, TNS, US] (Hale 1965: 286, as Parmelia); 14 km west of San Cristóbal, Hale 20566 [US] (Hale 1965: 286, as Parmelia); (Coutiño \& Mojica 1982: 172). — (2) Wolf \& Sipman 1814; (7) Wolf \& Sipman 2264; Breedlove \& Bourell 67289; Breedlove 58376.

Parmotrema cristiferum (Tayl.) Hale

El Suspiro, 10 km N of Berriozábal, Hale 20220 [US] (Hale 1965: 242, as Parmelia); El Sumidero, near Tuxtla Gutiérrez, Hale 20073 [S, TNS, US] (Hale 1965: 242, as Parmelia); Hacienda, Matuda s.n. [MICH] (Hale 1965: 242, as Parmelia). - Breedlove \& Bourell 67677, 67695, 67696; Breedlove 68759. NB. The Breedlove specimens have an additional TLC spot similar to gyrophoric acid; medulla C-.

Parmotrema dilatatum (Vain.) Hale

Just south of Teopisca, Hale 20522 [COLO, DUKE, LISU, MSC, REN, S, TNS, US] (Hale 1965: 246, as Parmelia); km 1145 on highway 190, west of San Cristóbal, Hale 20202 [COLO, US] (Hale 1965: 246, as Parmelia); 18 km southeast of San Cristóbal, Hale 20274 [US], 20250 [LISU, US] (Hale 1965: 246, as Parmelia); Lagos de Monte Bello, Hale 20413 [COLO, US] (Hale 1965: 246); Mt. Ovando, Matuda 28 [TNS] (Hale 1965: 246, as Parmelia). N.B! all sensu Hale 1965, incl. P. robustum (Degel.) Hale. - Breedlove \& Bourell 68522 (TLC: with echinocarpic acid).

Parmotrema disparile (Zahlbr.) Hale

El Sumidero, near Tuxtla Gutiérrez, Hale 20078 [DUKE, MSC, REN, S, TNS, US] (Hale 1965: 248, as Parmelia); road to El Suspiro, north of Berriozábal, Hale 20081, 20115 [US] (Hale 1965: 248, as Parmelia).

Parmotrema dominicanum (Vain.) Hale

$2 \mathrm{~km}$ east of Oaxaca-Chiapas State line, on highway 190, Hale 19888 [S, US] (Hale 1965: 249, as Parmelia); km. 956 on highway 190, west of Ocozocoautla, Hale 20596 [US] (Hale 1965: 249, as Parmelia). 
Parmotrema eciliatum (Nyl.) Hale

(2) Wolf \& Sipman 1648, 1813; (3) Wolf \& Sipman 1977.

Parmotrema endosulphureum (Hillm.) Hale

50 km west of Tuxtla Gutiérrez, Hale 19941 [DUKE, MSC, REN, S, TNS, US] (Hale 1965: 251, as Parmelia).

Parmotrema eurysacum (Hue) Hale

Breedlove 68994, 70640.

Parmotrema flavescens (Kremp.) Hale

Breedlove \& Bourell 67115; Breedlove 58366b, Breedlove 70239.

Parmotrema hababianum (Gyelnik) Hale Just south of Teopisca, Hale 20499 [S, US] (Hale 1965: 326, as Parmelia); 2 km north of highway 190 on road to Puebla Nueva, Hale 20164 [US] (Hale 1965: 327, as Parmelia); both cryptochlorophaeic acid-strain. - Breedlove \& Bourell 67686 (TLC: norlobaridone).

Parmotrema latissimum (Fée) Hale

El Sumidero, near Tuxtla Gutiérrez, Hale 20187 [MSC, US] (Hale 1965: 254, as Parmelia); 50 km west of Tuxtla Gutiérrez, Hale 21050 [DUKE, US] (Hale 1965: 254, as Parmelia); El Suspiro, 9 km north of Berriozábal, Hale 21232 [US] (Hale 1965: 254, as Parmelia). - Breedlove \& Bourell 67630.

Parmotrema leucosemothetum (Hue) Hale

40 km southeast of Comitán, Hale 20484 [US], 20483 [COLO, US] (Hale 1965: 331, as Parmelia); 14 km west of San Cristóbal, Hale 20573 [US] (Hale 1965: 331, as Parmelia). — (10) Wolf 1710.

Parmotrema mellissii (Dodge) Hale Lagos de Monte Bello, Hale 20961 [DUKE, MSC, REN, TNS, US] (Hale 1965: 298, as Parmelia); El Suspiro, 10 km north of Berriozábal, Hale 20235 [S, US] (Hale 1965: 298, as Parmelia). - (3) Wolf \& Sipman 1991; Breedlove \& Bourell 67357A; Breedlove 57427.

Parmotrema mesogenes (Nyl.) Hale

El Sumidero, Tuxtla Gutiérrez, Hale 20026 [US], 19909 [US] (Hale 1965: 256, as Parmelia); Mt. Ovando, Apr 1936, Matuda s.n. [MICH holotype, US] (Hale 1965: 250, as Parmelia ebulliens). - Breedlove \& Bourell 67462c.

Parmotrema mirandum (Hale) Hale

$8 \mathrm{~km}$ east of Teopisca, Hale 20346 [US] (Hale 1965: 274, as Parmelia); $2 \mathrm{~km}$ north of highway 190 on road to Puebla Nueva, Hale 20165 [S, US] (Hale 1965: 274, as Parmelia).

Parmotrema mordenii (Hale) Hale

West of Ocozoautla, Hale 20612 [US] (Hale 1971: 20, as Parmelia).

Parmotrema myelochroum (Hale) Hale

50 km west of Tuxtla Gutiérrez, in deciduous mist forest, 1040 m, 18 Mar 1960, Hale 21049 [US holotype, TNS] (Hale 1965: 255, as Parmelia); 50 km west of Tuxtla Gutiérrez, Hale 20214 [US] (Hale 1965: 255, as Parmelia). - (7) Wolf \& Sipman 2241; Breedlove \& Bourell 68519, 68540; Breedlove 70288.

Parmotrema neotropicum Kurok. = Rimeliella neotropica.

Parmotrema peralbidum (Hale) Hale

Lagos de Monte Bello, Hale 20397 [US] (Hale 1965: 258, as Parmelia).

Parmotrema praesorediosum (Nyl.) Hale

El Zapotal, Tuxtla Gutiérrez, Hale 19993 [S, US] (Hale 1965: 259, as Parmelia). 
Parmotrema rampoddense (Nyl.) Hale

$2 \mathrm{~km}$ north of highway 190 on road to Puebla Nueva, Hale 20171, 20185 [US] (Hale 1965: 305, as Parmelia); El Zapotal, Tuxtla Gutiérrez, Hale 20004 [US] (Hale 1965: 305, as Parmelia). - Breedlove \& Bourell 67462b; Breedlove 57004.

Parmotrema robustum (Degel.) Hale

(1) Wolf \& Sipman 1741, 1783, 1785; (2) Wolf \& Sipman 1863, 1866; (5) Wolf \& Sipman 2140; Breedlove \& Bourell 68329, 68417, 68491; Breedlove 57442, 69772.

Parmotrema sancti-angelii (Lynge) Hale Breedlove 70640a.

Parmotrema stuppeum (Tayl.) Hale

14 km west of San Cristóbal, Hale 20543 [MSC, REN, US] (Hale 1965: 309, as Parmelia).

Parmotrema subarnoldii (des Abb.) Hale

50 km west of Tuxtla Gutiérrez, Hale 19904 [US], 19935 [DUKE, REN, S, US] (Hale 1965: 310, as Parmelia).

Parmotrema subrugatum (Kremp.) Hale

El Suspiro, $10 \mathrm{~km} \mathrm{~N}$ of Berriozábal, Hale 20207 [US] (Hale 1965: 342, as Parmelia). -

(3) Wolf \& Sipman 1909 [ECOSUR].

Parmotrema tinctorum (Nyl.) Hale

South of Teopisca, Hale 20520 [S, TNS, US] (Hale 1965: 265, as Parmelia); El Sumidero, Tuxtla Gutiérrez, Hale 21210 [US] (Hale 1965: 265, as Parmelia). - (7) Wolf \& Sipman 2208; Breedlove \& Bourell 67113, 67377, 67428a, 68100, 68225, 68237, 68536; Breedlove 58378.

Parmotrema ultralucens (Krog) Hale

8 km east of Teopisca, Hale 20335 [S, US] (Hale 1965: 311, as Parmelia subcrinita); Hacienda, Matuda s.n. [MICH] (Hale 1965: 311, as Parmelia subcrinita). - Breedlove \& Bourell 67163, 68121; Breedlove 58377.

Parmotrema viridiflavum (Hale) Hale

$50 \mathrm{~km}$ southeast of Comitán, Lagos de Monte Bello, mature pine forest, $1220 \mathrm{~m}$, Hale 20415 [US holotype, DUKE, REN, S, TNS] (Hale 1965: 275, as Parmelia).

Parmotrema xanthinum (Müll. Arg.) Hale

Hacienda, Matuda s.n. [MICH] (Hale 1965: 270, as Parmelia aberrans); 8 km east of Teopisca, Hale 20355 [DUKE, S, TNS, US] (Hale 1965: 270, as Parmelia aberrans, Almeda \& Dey 1973: 542, as Parmelia madagascariacea). (Protolichesterinic acid only:) 2 km north of highway 190 on road to Puebla Nueva, Hale 20163 [US] (Hale 1965: 276, as Parmelia); km 956 on highway 190, west of Ocozocoautla, Hale 20594 [US] (Hale 1965: 276, as Parmelia). (Protolichesterinic acid + unkn. KC+ subst.:) El Zapotal, Tuxtla Gutiérrez, Hale 19975 [US] (Hale 1965: 277, as Parmelia). - (5) Wolf \& Sipman 2127; Breedlove \& Bourell 67111, 67162, 67462d, 67479.

Parmotrema zollingeri (Hepp) Hale

$2 \mathrm{~km}$ north of highway 190 on road to Puebla Nueva, Hale 20186 [COLO, DUKE, LISU, MSC, REN, S, TNS, US] (Hale 1965: 268, as Parmelia); km 916 on highway 190, west of Ocozocoautla, Hale 20600 [TNS, US] (Hale 1965: 268, as Parmelia). - (7) Wolf \& Sipman 2239; Breedlove \& Bourell 67428; Breedlove 70638, 70657.

Parmotremopsis antillensis (Nyl.) Elix \& Hale

Hale 20238 [US] (Hale 1976b: 19, as Parmelina). 
Parmotremopsis phlyctina (Hale) Elix \& Hale Hale 20202 [S, US] (Hale 1976b: 40, as Parmelina).

Peltigera austroamericana Zahlbr.

(2) Wolf \& Sipman 1831; Breedlove 51829, 57077.

Peltigera canina (L.) Willd.

Monte Ovando, Matuda (González de la Rosa \& Guzmán 1976: 42). The delimitation of this species has been much restricted in recent times, and Chiapas records are likely to be $P$. austroamericana.

Peltigera collina (Ach.) Schrad.

(1) Wolf \& Sipman 1736a; Breedlove 57432.

Peltigera polydactyla (Neck.) Hoffm. Lagos de Monte Bello, Guzmán 10771 (González de la Rosa \& Guzmán 1976: 44); Monte Ovando, Matuda s.n. (González de la Rosa \& Guzmán 1976: 44). Like P. canina, this species has undergone a change in delimitation and is now considered unlikely to occur in Chiapas. The material may belong to $P$. dolichorrhiza.

Phaeographina caesiopruinosa (Fée) Müll. Arg. El Suspiro, Hale 20133, 20215 [US] (Wirth \& Hale 1963: 102); West of Tuxtla Gutiérrez, Hale 19903 [US] (Wirth \& Hale 1963: 102). — (7) Wolf \& Sipman 2194a.

Phaeographina chrysocarpa (Raddi) Redgr. El Suspiro, Hale 20173 [S, TNS, US] (Wirth \& Hale 1963: 102).

Phaeographina elliptica Wirth \& Hale North of Berriozábal, 22 Mar 1960, Hale 20088 [US holotype] (Wirth \& Hale 1963: 104).

Phaeographina scalpturata Auct. West of Tuxtla Gutiérrez, Hale 19898 [US] (Wirth \& Hale 1963: 104). — (7) Wolf \& Sipman 2194, 2257, 2277 [ECOSUR].

Phaeographina strigops Wirth \& Hale Lagos de Monte Bello, 25 Mar 1960, Hale 20383 [US holotype] (Wirth \& Hale 1963: 104).

Phaeographis dendritica (Ach.) Müll. Arg. West of Tuxtla Gutiérrez, Hale 19895 [US] (Wirth \& Hale 1963: 105).

Phaeographis exaltata (Mont. \& v.d. Bosch) Müll. Arg. Lagos de Monte Bello, Hale 20394 [US] (Wirth \& Hale 1963: 106). — (7) Wolf \& Sipman 2234.

Phaeographis sericea (Eschw. in Mart.) Müll. Arg. North of Berriozábal, Hale 20107 [S, US] (Wirth \& Hale 1963: 106).

Phaeophyscia endococcinodes (Poelt) Essl.

(1) Wolf \& Sipman 1738, 1750.

Phaeophyscia hirsuta (Mereschk.) Moberg

(4) Wolf \& Sipman 2022.

Phaeophyscia hispidula (Ach.) Moberg

(1) Wolf \& Sipman 1752; Breedlove \& Bourell 67689, 67694, 67712, 67717.

Phaeophyscia limbata (Poelt) Kashiw.

Breedlove 57409.

Phyllobaeis erythrellus (Mont.) Kalb

Breedlove 57080.

Phyllopsora chlorophaea (Müll. Arg.) Zahlbr.

(5) Wolf \& Sipman 2094. 
Phyllopsora cuyabensis (Malme) Zahlbr.

(1) Wolf \& Sipman 1758; Breedlove \& Bourell 68525.

Phyllopsora furfuracea (Pers.) Zahlbr.

Lagos de Monte Bello, near Cinco Lagunas, 1600 m, 14 May 1988, Breedlove \& Bourell

67771 [CAS] (Brako 1991: 46). - Breedlove \& Bourell 67771.

Physcia atrostriata Moberg

Breedlove \& Bourell 67682e.

Physcia dubia (Hoffm.) Lett.

(4) Wolf \& Sipman 2025.

Physcia integrata Nyl.

Breedlove \& Bourell 67371a, 67682a.

Physcia lacinulata Müll. Arg.

(5) Wolf \& Sipman 2117; (7) Wolf \& Sipman 2213.

Physcia sorediosa (Vain.) Lynge

(1) Wolf \& Sipman 1754; (4) Wolf \& Sipman 2033 [ECOSUR]; Breedlove \& Bourell 67682.

Piccolia conspersa (Fée) Hafellner

(1) Wolf \& Sipman 1803 [ECOSUR].

Placopsis parellina (Nyl.) Lamb

Breedlove 58171.

Polymeridium proponens (Nyl.) R. C. Harris

(5) Wolf \& Sipman 2139.

Porina heterospora (Fink) R. C. Harris

(1) Wolf \& Sipman 1747; (3) Wolf \& Sipman 1957 [ECOSUR], 1968. The spores are fusiform-clavate and measure c. $90 \times 18 \mathrm{~mm}$, including a thick gelatinous sheat and are about 14-celled.

Porina cf. mastoidea (Ach.) Müll. Arg.

(5) Wolf \& Sipman 2067, 2090, 2091, 2121. The taxonomy of this species and its relatives seems still unclear, therefore the identification is provisional.

Porina nucula Ach.

(7) Wolf \& Sipman 2267.

Pseudevernia consocians (Vain.) Hale \& W. Culb.

(2) Wolf \& Sipman 1901; (4) Wolf \& Sipman 2031a [ECOSUR]; Breedlove \& Bourell 68317, 68327, 68334, 68414.

Pseudevernia intensa (Nyl.) Hale \& W. Culb.

(2) Wolf \& Sipman 1907; (3) Wolf \& Sipman 1970; Breedlove \& Bourell 67171, 67263A.

Pseudocyphellaria aurata (Ach.) Vain.

Breedlove 57436, 58098.

Pseudocyphellaria clathrata (De Not.) Malme

Breedlove 71351.

Pseudocyphellaria crocata (L.) Vain.

(3) Wolf \& Sipman 2012.

Pseudoparmelia amazonica (Nyl.) Hale = Canoparmelia amazonica.

Pseudoparmelia caroliniana (Nyl.) Hale = Canoparmelia caroliniana.

Pseudoparmelia salacinifera (Hale) Hale = Canoparmelia salacinifera . 
Pseudoparmelia cubensis (Nyl.) Elix \& Nash

El Sumidero Canyon, N of Tuxtla Gutiérrez, Hale 20030 [US] (Hale 1976a: 50, as Pseudoparmelia sphaerospora; Elix \& Nash 1998: 490).

Pseudoparmelia sphaerospora auct., non (Nyl.) Hale = Pseudoparmelia cubensis.

Pseudoparmelia texana (Tuck.) Hale = Canoparmelia texana.

Pseudoparmelia uleana (Müll. Arg.) Elix \& Nash

$2 \mathrm{~km} \mathrm{~N}$ of Highway 190 on road to Puebla Nueva, W of Chiapas, Hale 20174 [US] (Elix

\& Nash 1998: 490).

Punctelia rudecta (Ach.) Krog

El Sumidero canyon, north of Tuxtla Gutiérrez, 1960, Hale 20019 [US] (Culberson 1962:

568, as Parmelia); west of Tuxtla Gutiérrez, 1960, Hale 20585 [US] (Culberson 1962:

568, as Parmelia). - (1) Wolf \& Sipman 1777; (2) Wolf \& Sipman 1903; (7) Wolf \& Sipman 2212.

Punctelia semansiana (W. Culb. \& C. Culb.) Krog

(2) Wolf 1647, 1893, 1904; Breedlove \& Bourell 68323, 68326, 68216; Breedlove 57085

(?, no conidia seen).

Punctelia subrudecta (Nyl.) Krog

(2) Wolf \& Sipman 1871; (11) Wolf 1677.

Pyrenula anomala (Ach.) Vain.

(7) Wolf \& Sipman 2199 [ECOSUR], 2235.

Pyrenula cruenta (Mont.) Vain.

(13) epífita colectada de 0-2 m, Wolf \& Chanona 2657 [ECOSUR].

Pyrgillus javanicus (Mont. \& v. d. Bosch) Nyl.

Lagunas de Montebello, Kalb \& Plöbst S.n. (Tibell 1996: 56).

Pyrrhospora russula (Ach.) Haf. in Kalb \& Haf.

(7) Wolf \& Sipman 2203; Breedlove \& Bourell 68255; Breedlove 58372.

Pyxine berteriana (Fée) Imshaug

Breedlove \& Bourell 67371.

Pyxine caesiopruinosa (Nyl.) Imshaug

Breedlove 58366.

Pyxine eschweileri (Tuck.) Vain.

(1) Wolf \& Sipman 1788.

Pyxine obscurascens Malme

Breedlove 58376 (8 km N of Ocozocoautla, 1065 m, 4 Feb. 1982).

Pyxine rhodesiaca Lynge

Breedlove 58367a.

Ramalina anceps Nyl.

(5) Wolf \& Sipman 2076.

Ramalina asahinae W. Culb. \& C. Culb.

$11 \mathrm{~km}$ w of San Cristóbal de las Casas, ca. 2154 m, Culberson 16538 [DUKE, holotypus]

(Culberson \& Culberson 1976: 374) (Culberson et al. 1990: 186) (Kashiwadani \& Kalb

1993: 8); Culberson 16539, 16540, 16541, 18193 [DUKE] (Culberson et al. 1990: 186).

Ramalina aspera Räs.

(11) Wolf 1685.

Ramalina celastri (Spreng.) Krog \& Swinsc.

Breedlove 57097, 58159, 58240. 
Ramalina cochlearis Zahlbr.

(3) Wolf \& Sipman 1983 [ECOSUR].

Ramalina peranceps Nyl.

(7) Wolf \& Sipman 2221.

Ramalina sayreana W. Culb. \& C. Culb.

$11 \mathrm{~km}$ W of San Cristóbal de las Casas, Culberson 16544 [DUKE] (Culberson et al. 1990: 185).

Relicina abstrusa (Vain.) Hale

Hale 20028 [S, US] (Hale 1975a: 17). — (5) Wolf \& Sipman 2081, 2154 [ECOSUR].

Rimelia cetrata (Ach.) Hale \& Fletcher

Breedlove 58302.

Rimelia diffractaica (Essl.) Hale \& Fletcher

Breedlove \& Bourell 67462.

Rimelia reticulata (Tayl.) Hale \& Fletcher

(1) Wolf \& Sipman 1776; (11) Wolf 1679; Breedlove \& Bourell 67677a, 68441; Breedlove 58376.

Rimelia simulans (Hale) Hale \& Fletcher

Lago de Monte Bello, Hale 20369, 20416, 20467 [US] (Hale 1971a: 33, as Parmelia); Breedlove 71357.

Rimelia subisidiosa (Müll. Arg.) Hale \& Fletcher

Breedlove \& Bourell 67114; Breedlove 70637.

Rimeliella neotropica (Kurok.) Kurok.

On conifers, km 1145 on highway 190, west of San Cristóbal, 2120 m, Hale 20190 [US holotype] (Kurokawa 1991: 6; Hale 1977: 438, as Parmotrema); Hale 20013, 21141, 20230, 20267, 20614, 21205 [US] (Hale 1977: 438, as Parmotrema; Kurokawa 1991: 6).

Rimeliella subcaperata (Kremp.) Kurok.

El Sumidero, Tuxtla Gutiérrez, Hale 20078a [US] (Hale 1965: 338, as Parmelia reparata).

Rimeliella subsumpta (Nyl.) Kurok.

14 km west of San Cristóbal, Hale 20568 [TNS, US] (Hale 1965: 316); south of Teopisca, Hale 20509 [DUKE, US] (Hale 1965: 316, as Parmelia, Kurokawa 1991: 9), Hale 20521 [US] (Kurokawa 1991: 9); El Sumidero Canyon, Tuxtla Gutiérrez, Hale 21044 [US] (Hale 1965: 316, as Parmelia, Kurokawa 1991: 9); 50 km west of Tuxtla Gutiérrez, Hale 19957 [US] (Hale 1965: 316, as Parmelia, Kurokawa 1991: 9). - (1) Wolf \& Sipman 1734; Breedlove \& Bourell 67462A, 67695a.

Rimeliella subtinctoria (Zahlbr.) Kurok. (salazinic acid)

El Sumidero, Tuxtla Gutiérrez, Hale 20013 [US] (Hale 1965: 318, as Parmelia); south of Teopisca, Hale 20503 [COLO, US] (Hale 1965: 318, as Parmelia); $50 \mathrm{~km}$ west of Tuxtla Gutiérrez, Hale 19921 [US] (no salazinic acid, "cryptochlorophaeic acid"; Hale 1965: 319, as Parmelia; Kurokawa 1991: 12). — Breedlove \& Bourell 67676.

Sarcographa tricosa (Ach.) Müll. Arg.

(7) Wolf \& Sipman 2231, 2250.

Solorina simensis Hochst. ex Flotow

(3) Wolf \& Sipman 2009.

Stereocaulon microcarpum Müll. Arg.

Breedlove 56764, 58162, 59886. The chemistry is not tested, therefore it can not be ruled out that $S$. pachycephalum Vain. is present. 
Stereocaulon myriocarpum Fr.

Breedlove 59957, 68820.

Stereocaulon strictum Th. Fr. var. compressum (Nyl.) Lamb ex Vezda

Breedlove 46261.

Sticta weigelii (Ach.) Vain.

(1) Wolf \& Sipman 1770; (3) Wolf \& Sipman 2008a; (5) Wolf \& Sipman 2039; Breedlove 57101, 57440, 57443, 58102, 58191, 71329.

Strigula nitidula Mont.

(2) Wolf \& Sipman 1825.

Tapellaria nana (Fée) R. Sant.

(2) Wolf \& Sipman 1827.

Teloschistes exilis (Michx.) Vain.

NE de San Cristóbal de las Casas, Nee \& Mor, 3498 [MEXU] (González de la Rosa

\& Guzmán 1976: 53); cerca de Sumidero, Laughin 98 (González de la Rosa \& Guzmán 1976: 53); Tuxtla Gutiérrez a San Cristóbal de las Casas, Brechtel s.n. [MEXU] (González de la Rosa \& Guzmán 1976: 53). - (4) Wolf 1700, Wolf \& Sipman 2015; Breedlove \& Bourell 67331, 68093, 68316; Breedlove 57095, 57405, 69875, 71472.

Teloschistes flavicans (Sw.) Norm.

Carretera Teapa a Bochil, cerca de Rayón, Guzmán 10429 (González de la Rosa \& Guzmán 1976: 54); O de San Cristóbal de las Casas, Guzmán 10636 (González de la Rosa \& Guzmán 1976: 54). - Breedlove \& Bourell 68158, 68450; Breedlove 57093, 58173, 58375, 69774.

Tephromela atra (Huds.) Haf.

(5) Wolf \& Sipman 2292; Breedlove \& Bourell 68520.

Thelotrema lacteum Kremp.

(13) epífita sobre tronco de Ceiba, colectada a $1.37 \mathrm{~m}$, Wolf \& Chanona 2622.

Thelotrema lepadinum (Ach.) Ach.

(3) Wolf \& Sipman 1944.

Thelotrema platycarpoides Tuck.

(7) Wolf \& Sipman 2268.

Thelotrema santense Tuck.

(6) Wolf \& Sipman 2167; Breedlove \& Bourell 68533 (sterile), from munic. Ocozocoautla, $830 \mathrm{~m}$.

Thelotrema spondaicum (Nyl.) Hale

Epífita sobre tronco de Ceiba, colectada a 1.28 m, Wolf \& Chanona 2611.

Thelotrema stylothecium Vain.

(2) Wolf \& Sipman 1855.

Thelotrema cf. subtile Tuck.

(2) Wolf \& Sipman 1857; (3) Wolf \& Sipman 1961.

Trapelia coarctata (Sm. \& Sow.) Choisy

(4) Wolf \& Sipman 2018.

Trypethelium nitidiusculum (Nyl.) R. C. Harris

(5) Wolf \& Sipman 2150.

Trypethelium tropicum (Ach.) Müll. Arg.

(7) Wolf \& Sipman 2202. 
Tylophoron protrudens Nyl.

(5) Wolf \& Sipman 2135.

Tylophoron vs. moderatum Nyl.

(7) Wolf \& Sipman 2259. Only sporodochia present.

Usnea angulata Ach.

Lagos de Montebello, Herrera \& Trappe s.n. [MEXU] (González de la Rosa \& Guzmán 1976: 57).

Usnea dasaea Stirt.

Tzentontwitz-Chalam, Herrera-Campos 3120 [MEXU] (Clerc \& Herrera-Campos 1997: 298).

Usnea strigosa (Ach.) Eaton

Monte Ovando, Matuda s.n. (González de la Rosa \& Guzmán 1976: 60).

Xanthoparmelia piedmontensis (Hale) Hale

Breedlove 58366c.

Xanthoparmelia somloensis (Gyelnik) Hale

Breedlove 69198, 69486.

Xanthoparmelia tasmanica (Hook. \& Tayl.) Hale

Breedlove 69198a.

Xanthopsora texana (W. A. Weber) G. Schneider \& W. A. Weber = Xanthopsorella texana.

Xanthopsorella texana (W. A. Weber) Kalb \& Hafellner

Road to el Suspiro, 5-7 km N of Berriozábal, Hale [M] (Schneider 1979: 160, as Xanthopsora). - Breedlove 70618.

\section{ACKNOWLEDGEMENTS}

El Colegio de la Frontera Sur (ECOSUR) is gratefully acknowledged for technical support for the fieldwork. Mrs. M. Bourell kindly provided material and further information about the Breedlove collections. Prof. T. Ahti (Helsinki, Finland) is gratefully acknowledged for providing determinations and confirmations of Cladoniaceae.

\section{REFERENCES}

Almeda, F. y J. P. Dey. 1973. Chemical and nomenclatural notes on the Parmelia xanthina complex. The Bryologist 76: 541-543.

Awasthi, D. D. 1975. A monograph of the lichen genus Dirinaria. Biblioth. Lichenol. 2, 108 pp., map 1-16, f. 1-59.

Brako, L. 1991. Phyllopsora (Bacidiaceae). Flora Neotropica Monograph 55: 1-66.

Breedlove, D. H. 1981. Flora of Chiapas. Part I. Introduction to the flora of Chiapas. The California Academy of Sciences Press. San Fransisco, California. 35 pp.

Breedlove, D. E. 1986. Listados florísticos de México. IV. Flora de Chiapas. Instituto de Biología, Universidad Nacional Autónoma de México. México, D.F. 246 pp.

Brodo, I. M. 1991. Studies in the lichen genus Ochrolechia. 2. Corticolous species of North America. Canad. Journ. Bot. 69(4): 733-772. 
Acta Botánica Mexicana (1998), 45: 1-29

Clerc, P. \& M. A. Herrera-Campos. 1997. Saxicolous species of Usnea subgenus Usnea (Lichenized Ascomycetes) in North America. Bryologist 100(3): 281-301.

Coutiño, B. \& A. Mojica. 1982. Estudio de líquenes corticícolas de bosque mesófilo de montaña y de coníferas del estado de Hidalgo. Bol. Soc. Mex. Mic. 17: 166-180.

Culberson, W. L. 1962. Some pseudocyphellate Parmeliae. Nova Hedwigia 4: 563-577, pl. 132-134.

Culberson, W. L. \& C. F. Culberson. 1976. Ramalina asahinae, a new boninic acid-producing species from Mexico. J. Jap. Bot. 51: 374-376.

Culberson, W. L. \& C. F. Culberson. 1981. The genera Cetrariastrum and Concamerella (Parmeliaceae): a chemosystematic synopsis. Bryologist 84: 273-314, fig. 1-30.

Culberson, C. F., W. L. Culberson \& A. Johnson. 1990. The Ramalina americana complex (Ascomycotina, Ramalinaceae): chemical and geographic correlations. The Bryologist 93: 167186.

Dávalos de Guzmán, L., F. Brizuela \& G. Guzmán. 1972. Estudios sobre los líquenes de México, I. Notas sobre algunas especies. Anal. Esc. Nac. Cienc. Biol. 19: 9-30.

Degelius, G. 1974. The lichen genus Collema with special reference to the extra-european species. Symb. Bot. Upsal. 20(2):1-215.

Delgadillo, M. C. \& A. Cárdenas S. 1989. Phytogeography of high-elevation mosses from Chiapas, Mexico. Bryologist 92(4): 461-466.

Elix, J. A. \& T. H. Nash. 1998. A monograph of the lichen genus Pseudoparmelia (Ascomycotina, Parmeliaceae). Bryologist 100(4): 482-498.

Esslinger, T. L. 1994. New species and new combinations in the lichen genus Physconia in North America. Mycotaxon 51: 91-99.

Flores V., O. \& P. Gerez. 1994. Biodiversidad y conservación en México. Vertebrados, vegetación y uso del suelo. Universidad Nacional Autónoma de México. México D.F. 439 pp.

Gierl, C. \& K. Kalb. 1993. Die Flechtengattung Dibaeis. Eine Übersicht über die rosafrüchtigen Arten von Baeomyces sens. lat. nebst Anmerkungen zu Phyllobaeis gen. nov. Herzogia 9: 593-645.

Godínez, J. L. \& M. M. Ortega. 1989. Liquenología de México, historia y bibliografía. Cuadernos del Instituto de Biología 3. México, D.F. 45 pp.

González de la Rosa, E. \& G. Guzmán. 1976. Estudios sobre los líquenes de México, III. Observaciones sobre especies no consideradas anteriormente. Bol. Soc. Mex. Mic. 10: 27-64.

Guzmán-Dávalos, L. \& I. Alvarez. 1987. Observaciones sobre los líquenes de Jalisco y de Chiapas. Rev. Mex. de Mic. 3: 217-230.

Hale, M. E. 1961. The occurrence of Lobaria amplissima (Hoffm.) Schreb. in tropical America. Lichenologist 1: 266-267.

Hale, M. E. \& S. Kurokawa. 1964. Studies on Parmelia, subgenus Parmelia. Contr. U.S. Natl. Herb. 36(4): 121-191, pl. 1-9, fig. 1.

Hale, M. E. 1965. A monograph of Parmelia subgenus Amphigymnia. Contr. U.S. Natl. Herb. 36(5): 193-358, pl. 1-16, f. 1-29.

Hale, M. E. 1971. Morden-Smithsonian Expedition to Dominica: The lichens (Parmeliaceae). Smithsonian Contr. Bot. 4: 1-25.

Hale, M. E. 1971a. Five new Parmeliae from tropical America. Phytologia 22: 30-35.

Hale, M. E. \& M. Wirth. 1971. Notes on Parmelia subgenus Everniiformes with descriptions of six new species. Phytologia 22: 36-40.

Hale, M. E. 1975. A revision of the lichen genus Hypotrachyna (Parmeliaceae) in tropical America. Smithsonian Contr. Bot. 25: i-iii, 1-73, f. 1-20.

Hale, M. E. 1975a. A monograph of the lichen genus Relicina (Parmeliaceae). Smithsonian Contr. Bot. 26: 1-32, fig. 1-16.

Hale, M. E. 1976. A monograph of the lichen genus Bulbothrix Hale (Parmeliaceae). Smithsonian Contr. Bot. 32: 1-29, f. 1-7.

Hale, M. E. 1976a. A monograph of the lichen genus Pseudoparmelia Lynge (Parmeliaceae). Smithsonian Contr. Bot. 31: 1-62, f. 1-18. 
Hale, M. E. 1976b. A monograph of the lichen genus Parmelina Hale (Parmeliaceae). Smithsonian Contr. Bot. 33: i-iii, 1-60, fig. 1-20.

Hale, M. E. 1977. New species in the lichen genus Parmotrema Mass. Mycotaxon 5: 432-448.

Harris, R. C. 1990. Some Florida lichens. Published by the author. New York. 109 pp.

Imshaug, H. A. 1956. Catalogue of Mexican lichens. Revue Bryol. Lichénol. 25: 321-385.

Imshaug, H. A. \& I. M. Brodo. 1966. Biosystematic studies on Lecanora pallida and some related lichens in the Americas. Nova Hedwigia 12: 1-59, pl. 1-10.

Kalb, K. 1982. (Schedae ad) Lichenes neotropici, Fascikel III (No. 81-120). Neumarkt/Opf., Germany. $12 \mathrm{pp}$.

Kalb, K. 1983. (Schedae ad) Lichenes neotropici, Fascikel VI (No. 201-250). Neumarkt/Opf., Germany. $16 \mathrm{pp}$.

Kalb, K. \& A. Vezda. 1988. Die Flechtengattung Mazosia in der Neotropis (eine taxonomischphytogeographische Studie). Folia Geobot. Phytotax. 23: 199-210.

Kalb, K. 1991. (Schedae ad) Lichenes neotropici, Fascikel XII (No. 476-525). Neumarkt/Opf., Germany. $16 \mathrm{pp}$.

Kashiwadani, H. \& K. Kalb. 1993. The genus Ramalina in Brazil. Lichenologist 25: 1-31.

Kurokawa, S. 1961. Anaptychiae (lichens) and their allies of Japan (6). J. Jap. Bot. 36: 51-66.

Kurokawa, S. 1962. A monograph of the genus Anaptychia. Beih. Nova Hedwigia 6: 1-115, pl. 1-9.

Kurokawa, S. 1973. Supplementary notes on the genus Anaptychia. Journ. Hattori Bot. Lab. 37: 563607.

Kurokawa, S. 1991. Rimeliella, a new lichen genus related to Rimelia of the Parmeliaceae. Annals Tsukuba Bot. Garden 10: 1-14.

Kurokawa, S. 1998. A catalogue of Heterodermia (Physciaceae). Folia Cryptog. Estonica 32: 21-25.

Marcano, V., A. Morales Méndez, H. Sipman \& L. Calderón. 1996. A first checklist of the lichen-forming fungi of the Venezuelan Andes. Tropical Bryology 12:193-235.

McVaugh, R 1987. Itinerary and gazetteer. In: Mickel J. T. (ed.). Liebmann's Mexican ferns. Contributions from the New York Botanical Garden 19: 5-38.

Rzedowski J. 1992. Diversidad y origen de la flora fanerogámica de México. In: Halffter G. (comp.). La diversidad biológica de Iberoamérica. Acta Zool. Mex. volumen especial. pp. 315-335.

Schneider, G. 1979. Die Flechtengattung Psora sensu Zahlbruckner - Versuch einer Gliederung. Biblioth. Lichenol. 13: 1-291, Fig. 50-84.

Sipman, H. J. M. 1983. A monograph of the lichen family Megalosporaceae. Biblioth. Lichenol. 18: 1-241, pl. 1-24.

Staiger, B. \& K. Kalb. 1995. Haematomma-Studien. Biblioth. Lichenol. 59: 1-222, Abb. 1-27.

Stenroos, S. 1989. Taxonomic revision of the Cladonia miniata group. Annales Bot. Fenn. 26(3): 237261.

Tibell, T. \& K. Kalb 1992. Calicium in the tropical and subtropical Americas. Nova Hedwigia 55: 1136.

Tibell, T. 1996. Caliciales. Flora Neotropica Monograph 69: 1-79.

Vainio, E. 1926. Lichenes Mexicani a F. M. Liebmann annis 1841- 1843 collecti, in Museo Hauniensi asservati. Dansk Bot. Arkiv 4(11): 1-25.

Wirth, M. \& M. E. Hale. 1963. The lichen family Graphidaceae in Mexico. Contr. U.S. Natl. Herb. 36(3): 63-119.

Yoshimura, I. \& A. J. Sharp. 1968. Some lichens from the Southern Appalachians and Mexico. The Bryologist 71(2): 108-113.

Yoshimura, I. 1995. The lichen genus Anzia (Parmeliaceae, Lecanorales) in Central and South America. In: Daniels, F. J. A., M. Schulz \& J. Peine (eds.). Flechten Follmann, Contributions to lichenology in honour of Gerhard Follmann. Koeltz, Koenigstein. pp. 377-387. 